\title{
Lack of general learning ability factor in a rat test battery measuring a wide spectrum of cognitive domains
}

\author{
Ferenc Kassai ${ }^{1,2}$, Aliz ]. Ernyey ${ }^{1,2, *}$, Kata Kozma ${ }^{1,2}$, Imola Plangár ${ }^{1,2}$, István Gyertyán ${ }^{1,2, *}$ \\ ${ }^{1}$ Department of Pharmacology and Pharmacotherapy, Semmelweis University, 1089 Budapest, Hungary \\ ${ }^{2}$ Institute of Cognitive Neuroscience and Psychology, Hungarian Academy of Sciences, 1117 Budapest, Hungary \\ *Correspondence: gyertyan.istvan@med.semmelweis-univ.hu (István Gyertyán); ernyey.aliz@med.semmelweis-univ.hu (Aliz ]. Ernyey)
}

DOI:10.31083/j.jin2101012

This is an open access article under the CC BY 4.0 license (https://creativecommons.org/licenses/by/4.0/).

Submitted: 30 April 2021 Revised: 15] June 2021 Accepted: 27 August 2021 Published: 28 January 2022

Objective: In the framework of a larger project aiming to test putative cognitive enhancer drugs in a system with improved translational validity, we established a rodent test battery, where different, clinically relevant cognitive domains were investigated in the same animal population. The aim of the current study was to check whether performances in the different tasks representing different cognitive functions are assay-specific or may originate in an underlying general learning ability factor. Methods: In the experiments 36 Long-Evans and 36 Lister Hooded rats were used. The test battery covered the following cognitive domains: attention and impulsivity (measured in the 5-choice serial reaction time task), spatial memory (Morris water-maze), social cognition (cooperation task), cognitive flexibility (attentional set shifting test), recognition memory (novel object recognition) and episodic memory (water-maze based assay). The outcome variables were analyzed by correlation analysis and principal component analysis (PCA). The datasets consisted of variables measuring learning speed and performance in the paradigms. From the raw variables composite variables were created for each assay, then from these variables a composite score was calculated describing the overall performance of each individual in the test battery. Results: Correlations were only found among the raw variables characterizing the same assay but not among variables belonging to different tests or among the composite variables. The PCAs did not reduce the dimensionality of the raw or composite datasets. Graphical analysis showed variable performance of the animals in the applied tests. Conclusions: The results suggests the assay outcomes (learning performance) in the system are based on independent cognitive domains.

\section{Keywords}

Cognition; $g$ factor; Principal component analysis; Population with widespread knowledge

\section{Introduction}

Diseases of the brain pose the greatest health care challenge of the near future [1]. Cognitive disorders make up a large part of the symptomatology, but their treatment is insufficient and the efficacy of the few available cognitive enhancer drugs is low. The high unmet medical need has continuously stimulated the development of novel drugs, however, in the last 18 years none of them passed the clinical tri- als, in most cases because of lack of efficacy. This failure suggests that the preclinical behavioral cognitive assays applied in drug development have low predictive value [2-5].

As different cognitive domains are impaired to a different extent in different cognitive disorders [6], a test battery, which intends to predict clinical efficacy, should preferably be composed of assays representing the vulnerable cognitive capabilities. With the aim to establish a rodent cognitive test battery with improved predictive power we constructed an assay system covering a wide spectrum of clinically relevant cognitive domains (see below). To mimic further the human circumstances, namely, the cognitive life experience of the subjects, the various learning tasks are taught to the same cohort of rats thereby a population with "widespread knowledge" is created. This population may then be transformed to various "patient populations" by applying distinct impairing methods and putative cognitive enhancers can subsequently be tested in these different "patient populations" [3]

In this paper we report studies with two cohorts of rats, one of the Long-Evans the other of the Lister hooded strain. We chose these strains as pigmented rats are known for their superior learning performance over white rats $[7,8]$. The cognitive domains in focus were modelled with the following assays: attention and impulsivity were tested in the 5-choice serial reaction time task (5-CSRTT) [9], rule learning and mental flexibility in the attentional set shifting task (ASST) [10], recognition memory in the novel object recognition test (NOR) [11] and spatial memory in the Morris water-maze test (MWM) [12]. To assess social cognition, particularly, cooperative task solving abilities, a novel cooperation task (COOP) was introduced [13]. Procedural learning and motor skills were tested in the in-house established pot jumping test (PJT) [14]. We also set up an episodic memory test (EPISM) by modifying the standard Morris water-maze procedure [15]. Beside the cognitive tasks, anxiety level of the animals was assessed in the elevated plus-maze (EPM) test [16].

Investigating the learning capabilities of the animals in our population with "widespread knowledge" raised the ques- 


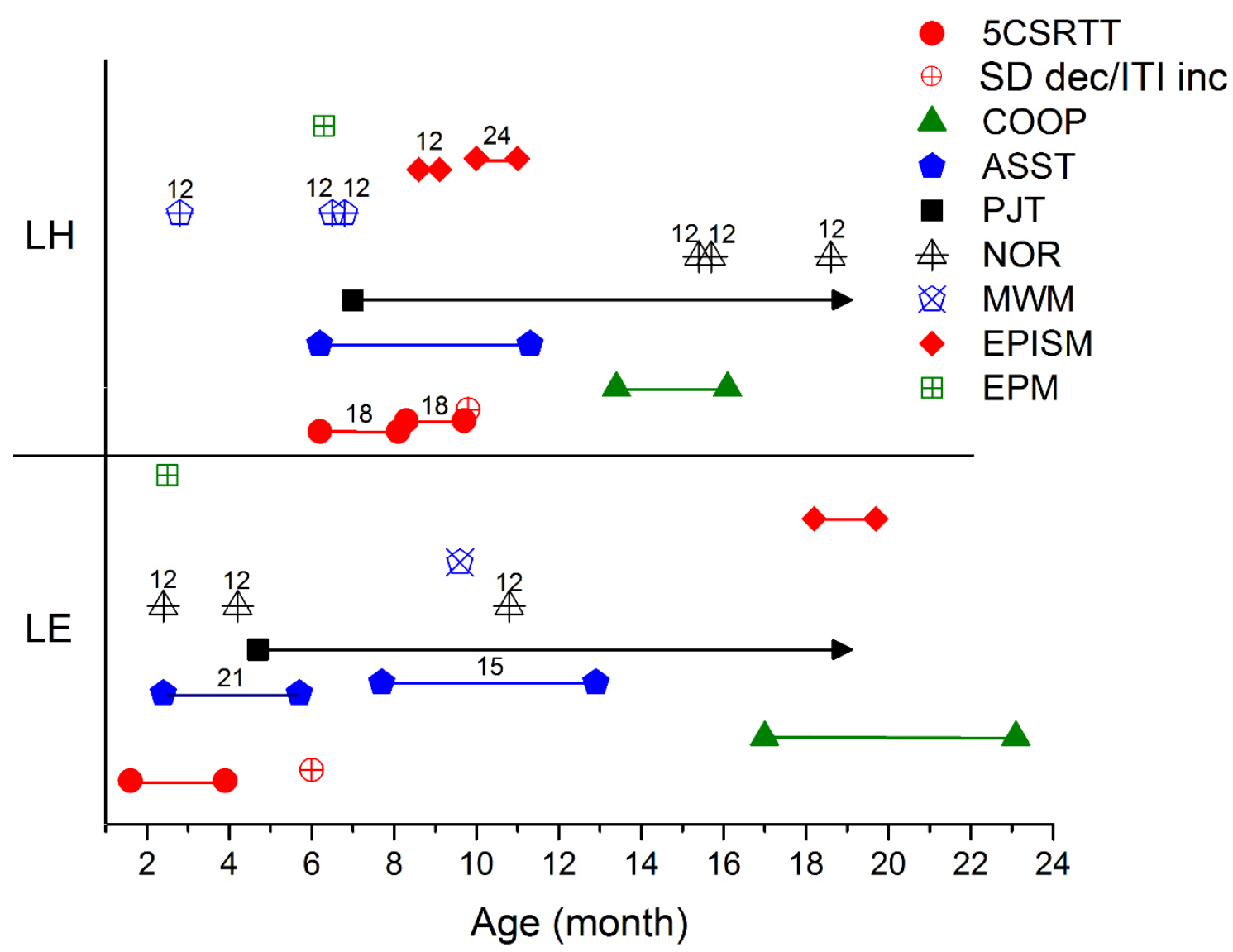

Fig. 1. Timing of the behavioral assays. Filled symbols indicate tests with long training duration (PJT test was run lifelong), while unfilled symbols indicate short duration tests. Numbers of animals are indicated above the symbols if not all of the rats in the same strain were tested at the same time. Upper panel Lister Hooded (LH), lower panel Long-Evans (LE) rats. (ASST, attentional set shifting task; COOP, cooperation task; 5-CSRTT, 5-choice serial reaction time task; EPISM, episodic memory test; EPM, elevated plus-maze; MWM, Morris water-maze test; NOR, novel object recognition test; PJT, pot jumping test. SD $\mathrm{dec} / \mathrm{ITI}$ inc indicates the two daily sessions, when task difficulty was increased by reducing stimulus duration (SD) or increasing inter-trial interval (ITI) (for details see chapter 'Behavioral assays'). After LE rats completed 5CSRTT training at the age of 4 months, they regularly participated in the test until the age of 6 months.

tion whether good performance is generalized or restricted to specific assays. Literature data of laboratory rodents indicate that a remarkable portion of the variance in performance displayed in different cognitive tasks can be explained by a general learning ability factor [17-25] akin to the human general intelligence factor ( $g$ factor, [26]). In the current study, we checked whether the cognitive performance of our rat populations was assay-specific or relied-at least partly-on a common $g$ factor.

\section{Materials and methods}

\subsection{Animals}

In the experiments two outbred strains were used: 36 male Lister Hooded [Charles River, Italy] (LH) and 36 male LongEvans [Janvier, France] (LE) rats. Animals were housed in groups of three in plastic cages (of $38 \times 23 \times 18 \mathrm{~cm}^{3}$ after arrival, and $43 \times 32 \times 21 \mathrm{~cm}^{3}$ later, when their body size required a larger cage) with wire grid top in a light controlled, sound attenuated room (reversed 12-h light/dark cycle, light on at 16:00 h). For environmental enrichment aspen bricks and cardboard tubes were placed in the cages. Daily food intake was limited to $40-45 \mathrm{~g}$ commercial pellet rat feed (ssniff $\mathrm{R} / \mathrm{M}+\mathrm{H}$ produced by Spezialdiäten $\mathrm{GmbH}$ (Soest, Germany) per cage. Animals were fed at the end of the active phase, 1-3 hours following the daily training. This feeding regime resulted in $85 \%$ (LE) and $90 \%$ (LH) body weight compared to ad libitum fed animals. The restricted food intake made the rats motivated to work for the reward ( $45 \mathrm{mg}$ purified dustless precision pellets, Bio-Serv, Flemington, NJ, USA) in the behavioral tasks. Access to tap water was ad libitum. Rats were intensively handled before and during their behavioral testing. Housing and all procedures carried out on animals were authorized by the regional animal health authority in Hungary (resolution number PEI/001/3572-4/2014) and conformed to the Hungarian welfare legislation, ARRIVE guidelines and the EU 63/2010 Directive. 


\subsection{Experimental design}

Behavioral assays were conducted between the age of 123 and 2-19 months of LE and LH rats, respectively (Fig. 1). For logistical reasons, in some of the assays not all the animals were trained at the same period of their life-span.

2.3 Behavioral assays

2.3.1 The 5-choice serial reaction time task paradigm (5-CSRTT)

The operant chamber (TSE, Germany) was equipped with five nose-poke modules. Animals were trained to nose-poke into a randomly chosen hole marked for $1 \mathrm{~s}$. In half of the animals, the 'classical' 5CSRTT paradigm was applied [9], where turning on the stimulus light served as a signal. For the rest of the population, a novel 5CSRTT method was used. In the latter case all the nose-poke modules were illuminated, and the signal was turning off the stimulus light. This arrangement originally aimed at testing the assumption that the detection of the 'off' signal is more difficult, therefore the attentional load is higher compared to the 'classical' 5CSRTT version. However, as it only took two days more to the animals to acquire the "off" version of the task [27], and afterwards the two groups showed similar performance, data of the two groups were pooled for the analyses.

In both paradigms, correct responses were rewarded with a pellet delivered into the magazine. Nose-poke into the magazine initiated the next trial. The animal made an incorrect response if nose-poked into one of the non-signaled holes, a premature response, if nose-poked into any of the holes during the 5 sec long inter-trial interval, and an omission, if it did not respond to the stimulus. Incorrect and premature responses as well as omissions were punished with a 5 sec timeout period (house light off). Duration of a daily test session was $20 \mathrm{~min}$. Proportion of correct responses and number of premature responses were regarded as measures of attention and impulsivity, respectively [9]. Rats were trained for the task in stages with gradually increasing difficulty. For the detailed training procedure see Supplementary materials.

When rats completed the training, task difficulty was increased in two ways. In one session, stimulus duration was decreased to $250 \mathrm{~ms}$, while in another session, inter-trial interval was increased to $8 \mathrm{~s}$. The first modification increases the attentional load of the task, while the second induces impulsive responses [9].

\subsubsection{Cooperation task in the Skinner box (COOP)}

The assay is described in detail in Kozma et al. [13]. Two rats were placed in the same Skinner box (MedAssociates, USA). The opposite walls of the chamber were equipped with one nose-poke module and one magazine for each. In order to obtain food reward, animals had to perform simultaneous nose-pokes after a stimulus light was turned on in both modules. The nose-pokes at the opposite sides were regarded as simultaneous, if the delay between them did not exceed $1 \mathrm{~s}$. Non-simultaneous responses or repeated nosepokes to the same module were punished with $5 \mathrm{~s}$ time-out. Rats were trained for the task in stages with gradually de- creasing intervals allowed for the "simultaneous" nose-pokes from $10 \mathrm{~s}$ ( $5 \mathrm{~s}$ in case of $\mathrm{LH})$ to $1 \mathrm{~s}$. The training was carried out in three pairings. First, two cage-mates were trained, which were unfamiliar with the task (naïve-naïve, $A+B$ ). After that, the third animal - naïve to the task - formed a pair with one of its experienced cage-mates (naïve-experienced, $\mathrm{C}+\mathrm{B}$ ). Finally, the last remaining combination of the cagemates worked together. Animals in these pairs (experiencedexperienced, $\mathrm{A}+\mathrm{C}$ ) had previously been trained for the task with one of their cage-mates. In case of LE, not all of the pairs were recruited from the same cage because some animals could not complete the training, died, or were aggressive with their pair (for details see Supplementary materials, section 'Handling of missing data').

\subsubsection{Attentional set shifting task (ASST)}

We applied a modified version of the task originally described by Birrell and Brown [10]. The experiment was conducted in a custom made $62 \times 54 \mathrm{~cm}^{2}$ box with $30 \mathrm{~cm}$ high walls. The box was divided by inner walls to a start area, a choice area and two reward areas. A rat was placed into the start area. The experimenter opened the door of the start area to allow the rat to enter the choice area. At the back wall of the choice area two openings led to the reward areas. The task of the rat was to find the correct entrance leading to that reward area where a pellet was hidden under sizzle nest material in a terra-cotta flowerpot. Three types of stimuli were used for signaling the correct entrance: odor cue placed above the entrances, floor texture at the step of the doors and color cues also placed at the top of the doors. Rats had to complete several learning stages. On a training day rats had 12 trials in the same training stage. Within a daily session, consecutive correct choices were progressively rewarded: after the 1 st, 2 nd and 3 rd correct choices rats could obtain 1 pellet, in the 4 th and 5th successful trials 2 pellets, and from the 6 th successful trial on 3 pellets. If a rat made an incorrect choice, the number of rewards was reset to 1 in the next trial. If a rat obtained 17 rewards in a daily session 3 times within a 4-day long time bin, it could advance to the next training stage. For detailed description of the stages see Supplementary materials.

\subsubsection{Morris water-maze test ( $M W M)$}

The task is based on the method described by Morris [12]. The task of the animals was to find a hidden $10 \mathrm{~cm}$ diameter platform in a $190 \mathrm{~cm}$ diameter, $60 \mathrm{~cm}$ deep circular tank filled with $39 \mathrm{~cm}$ water $\left(23 \pm 1^{\circ} \mathrm{C}\right)$. The platform was placed $0.5 \mathrm{~cm}$ under the water surface, in the south-east quadrant, at about $40 \mathrm{~cm}$ distance from the side wall of the pool. Extra-maze cues were placed on the wall of the experimental room, in order to facilitate the orientation during swimming. Animals were trained on four consecutive days in three daily trials with $30 \mathrm{~min}$ inter-trial intervals. They were placed into the water at the north, east, south or west edge of the pool in systemic rotation and were given 180 
$s$ to escape onto the hidden target. Movement of animals was recorded with Smart v3.0 video tracking system software (Panlab, Barcelona, Spain).

\subsubsection{Episodic memory in Morris water-maze (EPISM)}

In the modified Morris water-maze navigation task, rats had to find the hidden platform by taking into account the actual time of the day [15]. The platform was placed into the north-west target zone at 6:00 AM ('morning') and into the south-west part of the maze at 2:00 PM ('evening'). A separation board (height $60 \mathrm{~cm}$ ) was inserted along the east-west diameter of the pool. Each animal was released at the 'east' starting point, where a $20 \mathrm{~cm}$ wide opening in the separation board provided transit between the two halves of the pool. Two trials were performed in each 'morning' and 'evening' session: a 'query' trial (trial 1) and a 'confirmatory 'trial (trial 2), 30 min apart. Swimming directly to the target was rewarded with three pellets. When rats searched for the platform in the incorrect half of the maze, they were allowed to correct their wrong choice by swimming to the platform through the opening. In this case they were not rewarded with food pellets.

\subsubsection{Novel object recognition test (NOR)}

The task is based on the method described by Ennaceur and Delacour [11]. The test was performed in a $48 \times 48$ $\times 42 \mathrm{~cm}^{3}$ test box, where behavior of animals was observed through a video camera system. Before the test, rats had been habituated to the test box for $3 \mathrm{~min}$. On the next day the acquisition trial was conducted, where two identical objects were placed into the box. Rats were allowed to investigate them for $3 \mathrm{~min}$. $80 \mathrm{~min}$ later, in the retention trial, one of the familiar objects and a novel object were placed into the box. Similarly to the acquisition trial, animals were allowed to explore the objects for $3 \mathrm{~min}$. Time spent with exploring the objects was recorded. Animals which explored the objects for less than $10 \mathrm{~s}$ in any of the trials were excluded from the experiment.

\subsubsection{Pot jumping test (PJT)}

The test was designed according to Ernyey et al. [14] and was intended to follow motor learning abilities of the rats. The experiment was conducted in the MWM tank, where 12 flower pots ( $16 \mathrm{~cm}$ high and $10 \mathrm{~cm}$ wide at the bottom) were placed upside down forming a circle. Distance between the adjacent pots gradually increased from $18 \mathrm{~cm}$ to $46 \mathrm{~cm}$. The tank was filled with $6 \mathrm{~cm}$ deep cold water to restrain rats climbing off the pots. During training, animals were placed onto the start pot, which was within the shortest distance from the next pot. They could freely move on the pots for $3 \mathrm{~min}$ and their behavior was observed and recorded with a video camera system. Trainings were repeated at least once in a month and the distance covered by the animals was recorded.

\subsubsection{Elevated plus-maze (EPM)}

The experiment was carried out in a custom made elevated plus maze according to Pellow et al. [16]. The maze consisted of two $50 \times 15 \mathrm{~cm}^{2}$ closed arms bordered with $40 \mathrm{~cm}$ high walls, and two open arms of the same size without border walls. The maze was $50 \mathrm{~cm}$ above the floor of the experiment room. Animals were placed into the center and they could freely move in the maze for $5 \mathrm{~min}$. Their behavior was observed through a video camera system. Time spent in the open and closed arms and arm entries were recorded.

\subsection{Statistical evaluation}

Data were analyzed in three steps with condensing the information content of the variables. In most of the assays, performance was measured by multiple raw variables. First, these data were subjected to correlation analysis and principal component analysis (PCA). At the second step, a composite variable was created from the raw variables for each assay and these composite variables were analyzed in a similar way. Finally, a composite score was calculated from the composite variables for each individual, which characterized the overall performance in the test battery. It was expected, that this information compressing procedure increases the chance to detect correlations among the performances in the different cognitive domains. Correlation analyses and principal component analyses were performed by the Statistica software (version 13.5.0.17,TIBCO Software Inc.,Palo Alto, CA, USA).

\subsubsection{The selected raw variables}

Two distinct types of variables were used to describe skillfulness of animals in the behavioral assays. One group of variables measured the speed of acquiring the paradigm, the other group of parameters characterized the final performance level of the animals in the task after they completed the training (see their list in Table 1). Raw data were analyzed with correlation and principal component analysis (for details see next section).

In case of 5-CSRTT data, variables characterizing the learning speed were calculated from the training stages. Each stage represented a learning level, which was expressed in percentage of the final stage. Learning speed was described by the number of training days required to complete half (50\%) of the stages and to complete the highest stage (100\%). A third learning speed parameter was the training stage of an animal reached at the time when the mean learning stage of the particular strain best approximated the $50 \%$ stage of the training (i.e., at the inflection point of the sigmoid learning curve). The parameters describing performance were: (1) the mean number of rewards collected in the basic paradigm with $1 \mathrm{~s}$ stimulus duration (average of the last two days preceding the days of challenges stimulus duration decrease and inter-trial interval increase), (2) the decrease in the proportion of correct responses induced by the reduction of stimulus duration and (3) the increase in the proportion of premature responses induced by the increase of the inter-trial interval (Table 1). 
Table 1. List and codes of raw variables measuring cognitive capabilities, and results in the two rat strains (non-standardized raw data).

\begin{tabular}{|c|c|c|c|c|c|}
\hline Test & Type & Code & Description & LE mean $\pm \mathrm{SD}$ & $\mathrm{LH}$ mean $\pm \mathrm{SD}$ \\
\hline \multirow{7}{*}{ 5CSRTT } & \multirow{4}{*}{ Learning speed } & 5Ch_Lev@50 & individual training stage (\%) when the group mean learning stage best & $51.8 \pm 20.1$ & $48.7 \pm 30.5$ \\
\hline & & & approximated $50 \%$ & & \\
\hline & & $5 \mathrm{Ch} \_\mathrm{Td} 50$ & number of training days required to complete half of the training stages & $11.7 \pm 2.8$ & $22.7 \pm 11.0$ \\
\hline & & 5Ch_Td100 & number of training days required to complete the entire training & $18.8 \pm 5.1$ & $28.3 \pm 11.7$ \\
\hline & \multirow{3}{*}{ Performance } & 5Ch_rew & number of rewards collected in the basic paradigm with $1 \mathrm{~s}$ stimulus duration & $72.3 \pm 23.5$ & $61.8 \pm 19.2$ \\
\hline & & 5Ch_cdec & decrease $(\%)$ in the correct responses at decreased stimulus duration & $33.8 \pm 16.4$ & $27.9 \pm 12.1$ \\
\hline & & 5Ch_pinc & increase (\%) in the premature responses at increased inter-trial interval & $33.1 \pm 14.3$ & $44.2 \pm 10.8$ \\
\hline \multirow{6}{*}{ COOP } & \multirow{4}{*}{ Learning speed } & Co_Lev@50 & individual training stage (\%) when the group mean learning stage best & $50.8 \pm 33.3$ & $60.0 \pm 17.2$ \\
\hline & & & approximated 50\% & & \\
\hline & & Co_Td50 & number of training days required to complete half of all the training stages & $9.5 \pm 2.9$ & $5.1 \pm 1.0$ \\
\hline & & Co_Td100 & number of training days required to complete the entire training & $12.7 \pm 4.2$ & $7.0 \pm 1.3$ \\
\hline & \multirow[t]{2}{*}{ Performance } & Co_rew & $\begin{array}{l}\text { number of rewards obtained when the animal reached the reward criteria in } \\
\text { the last training stage }\end{array}$ & $52.0 \pm 6.0$ & $50.9 \pm 5.0$ \\
\hline & & Co_SuccTr & $\begin{array}{l}\text { proportion of successful trials (\%) when the animal reached the reward } \\
\text { criteria in the last training stage }\end{array}$ & $59.3 \pm 6.0$ & $58.7 \pm 4.7$ \\
\hline \multirow{2}{*}{ ASST } & \multirow{2}{*}{ Learning speed } & A_TdCD & number of training days required to complete compound discrimination & $46.4 \pm 20.8$ & $33.8 \pm 27.1$ \\
\hline & & A_Stage & rank of the highest stage reached & $2.1 \pm 1.8$ & $3.2 \pm 2.4$ \\
\hline \multirow{3}{*}{ MWM } & \multirow{3}{*}{ Learning speed } & M_day1 & mean escape latency (s) on the 1 st training day & $94.6 \pm 39.6$ & $113.6 \pm 40.3$ \\
\hline & & M_day2 & mean escape latency (s) on the 2 nd training day & $25.4 \pm 27.8$ & $36.8 \pm 31.9$ \\
\hline & & M_day3 & mean escape latency (s) on the 3 rd training day & $13.4 \pm 20.6$ & $16.5 \pm 23.0$ \\
\hline \multirow{4}{*}{ EPISM } & \multirow{2}{*}{ Learning speed } & E_first_tr1 & mean escape latency $(\mathrm{s})$ in the first 4 training sessions in trial 1 & $77.6 \pm 39.5$ & $46.4 \pm 20.5$ \\
\hline & & E_first_tr2 & mean escape latency $(\mathrm{s})$ in the first 4 training sessions in trial 2 & $35.3 \pm 25.2$ & $25.6 \pm 14.3$ \\
\hline & \multirow{2}{*}{ Performance } & E_last_tr1 & mean escape latency $(\mathrm{s})$ in the last 4 training sessions in trial 1 & $14.2 \pm 8.7$ & $15.2 \pm 9.8$ \\
\hline & & E_last_tr2 & mean escape latency $(\mathrm{s})$ in the last 4 training sessions in trial 2 & $12.6 \pm 10.2$ & $12.1 \pm 3.9$ \\
\hline NOR & Performance & NOR & discrimination index & $0.25 \pm 0.3$ & $0.14 \pm 0.4$ \\
\hline
\end{tabular}

In case of COOP assay, for all parameters, the values of a rat obtained in the two pairings (e.g., $\mathrm{A}+\mathrm{B}$ and $\mathrm{A}+\mathrm{C}$ in case of animal A) were averaged. Learning speed parameters were the same as in the 5-CSRTT (number of training days required to complete the half and all of the training stages, training stage reached at the time when the mean learning stage of the particular strain best approximated 50\%). Two performance parameters were applied: number of rewards obtained and the proportion of successful trials (\%) (Table 1). Both variables were calculated by averaging the values obtained on the day when the pair reached the reward criteria in the last training stage and on the two consecutive days. As an animal was trained in two pairings, six values were averaged.

Only few rats were able to complete the entire ASST training in both strains. According to their achieved stages a performance level score was assigned to each animal depending on how many cognitive functions it could use: 0-random choice; 1-discrimination only; 2-intra dimensional shift (IDS) or reversal (IDR); 3-IDS and reversal (IDR); 4-extra dimensional shift (EDS); 5-second EDS, back to the original relevant stimulus set (LE) or to a third stimulus set (LE, LH); 6-multiple EDS (LE) or EDS trials without stimulus substitution (LH) (see Supplementary materials for detailed description). The training days required to complete com- pound discrimination, and the final performance level score were used as learning parameters (Table 1).

In the MWM, all of the animals learned the position of the platform by the last experimental day. Their escape latencies were low and homogenous (Mean \pm SEM LE: $14.9 \pm 3.1 \mathrm{~s}$ LH: $9.6 \pm 1.4 \mathrm{~s}$ ). Therefore, in case of this test, performance parameters were not used. For describing learning speed, latencies on the first three training days were included in the analysis (Table 1).

In analysis of EPISM data, mean of the first trial escape latencies in the first four training sessions and mean of the second trial escape latencies in the first four training sessions were used as learning speed parameters. In this period, when animals were learning the platform locations, steeper decrease in the escape latencies (i.e., faster learning) resulted in lower average value. In the last four sessions, animals demonstrated stabilized performance, their escape latencies reflected the efficacy of mastering the task. Therefore, means of the first and second trial escape latencies in the last four sessions were used as performance parameters (Table 1).

In NOR test, discrimination index was calculated from the exploration times of novel and familiar objects in the retention trial by the following formula:

$$
\left[\left(\mathrm{t}_{\text {novel }}-\mathrm{t}_{\text {familiar }}\right) /\left(\mathrm{t}_{\text {novel }}+\mathrm{t}_{\text {familiar }}\right)\right] \times 100
$$


Discrimination index is a measure of recognition memory in the test (Table 1). Beside this parameter, total time spent on investigating the objects in the first and second trial were included in the analysis as parameters describing exploratory behavior (Table 2).

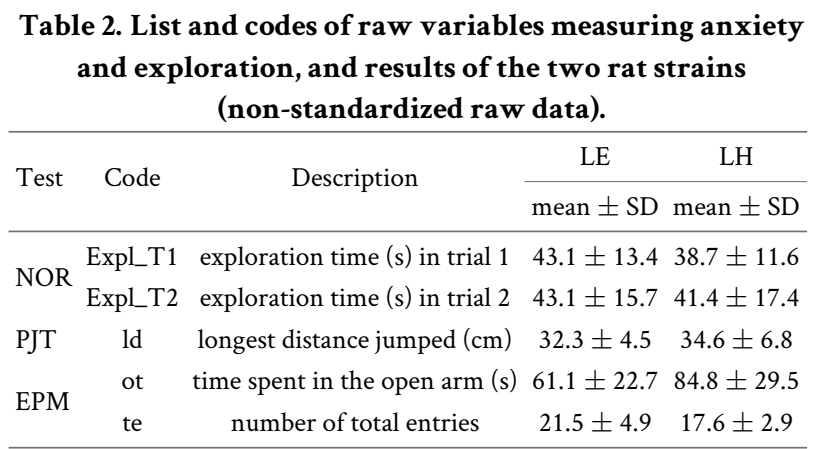

In case of the PJT test, the longest distance jumped over between two pots during the entire training period (personal best performance) was included in the dataset (Table 2). This parameter measures skillfulness and exploratory inclination.

From EPM data, the sum of closed and open arm entries and time spent in the open arm were involved in the analysis as variables of exploratory behavior (Table 2).

Handling of missing data is described in Supplementary materials (number of missing data were as follows:

5-CSRTT: 1 LE rat, ASST: 11 LE and 7 LH rats, COOP: 5 LE and 6 LH rats, EPISM: 1 LE rat, NOR: 8 LH rats).

\subsubsection{Analysis of raw variables}

Datasets of the two rat strains were analyzed separately. In the raw datasets, data were standardized by variance to make variables measured on different scale comparable.

This was followed by a correlation analysis where Pearson correlation coefficients were calculated. To reduce false discovery rate and to keep the $5 \%$ significance limit, sequential Bonferroni adjustment was applied [28].

Datasets were also analyzed with PCA in which the number of significant components in the model was determined by v-fold cross validation method [29]. As the PCA models could explain only a minor portion of the entire variance, a threshold value was introduced for the interpretation of PCA results. A variable was considered relevant along a component, if its loading was higher than 0.6 or lower than -0.6 (i.e., when it was in at least 0.6 units distance from the point of intersection along one of the components on the scatterplot). This way, the weak and therefore irrelevant linkages among variables were excluded.

\subsubsection{Calculation and analysis of composite variables}

The outcome of a PCA is more robust with higher subject to variable ratio [30]. To achieve this state we reduced the number of variables-while preserving the information stored in the raw data - by creating composite vari- able datasets from the raw standardized datasets. A composite variable was calculated for each test by summing up the values of the raw variables according to their valence in describing the effectiveness in the particular test (e.g., in case of 5-CSRTT composite variable, learning performance level reached at completion of the training was entered with a plus sign, while learning time until reaching half of the training stages was entered with a minus sign). Furthermore, an additional composite variable describing exploratory inclination (EXPL) was created by summing the longest distance reached in pot jumping, open time and total entries registered on the EPM and exploration time in trial one and two in the NOR test. In case of the NOR test, discrimination index was used as a single variable describing cognitive performance. After the calculations, data in the composite variable datasets were again standardized by variance.

The analysis of composite variables was identical to the analysis of raw variables (correlation analysis, PCA).

\subsubsection{Calculation and analysis of composite score}

To characterize the overall performance of an animal with a single number, a composite score was calculated for each individual by summing the values of the composite variables and NOR discrimination index.

Distribution of composite scores was investigated with histograms and normal probability plots. Mean and 95\% confidence interval was calculated for the strains. To examine, whether the composite score reflects a general success in all tests (i.e., whether animals with low composite score have low success in all tests and vice versa), animals were ranked according to their composite scores, and their composite variable values that describe their success in certain tests were plotted on a heat map.

\section{Results}

\subsection{Relation of the raw data variables}

The mean and standard deviation of all unstandardized raw variables are shown in Tables 1,2. In LH rats, correlations were found only between those variables that belonged to the same behavioral task. Similar pattern can be observed in LE rats, but in this dataset significant correlation was detected between the number of rewards collected in the cooperation task and time spent in the open arm in the EPM test $(r=0.49)$ (Fig. 2).

In the 5-CSRTT assay, significant correlations were detected among variables describing learning speed. In LE rats, training stage reached when mean learning stage of the strain best approximated $50 \%$ correlated with the number of training days required to complete the half and the entire training $(r=-0.87$ and -0.47 , respectively). Training days required to complete the half and the entire training also correlated $(r=$ 0.58). In LH rats only these two variables displayed correlation $(r=0.99)$. In the latter strain rewards collected in the basic paradigm and decrease in the number of rewards due to the reduction of stimulus duration also correlated $(r=0.52)$. 


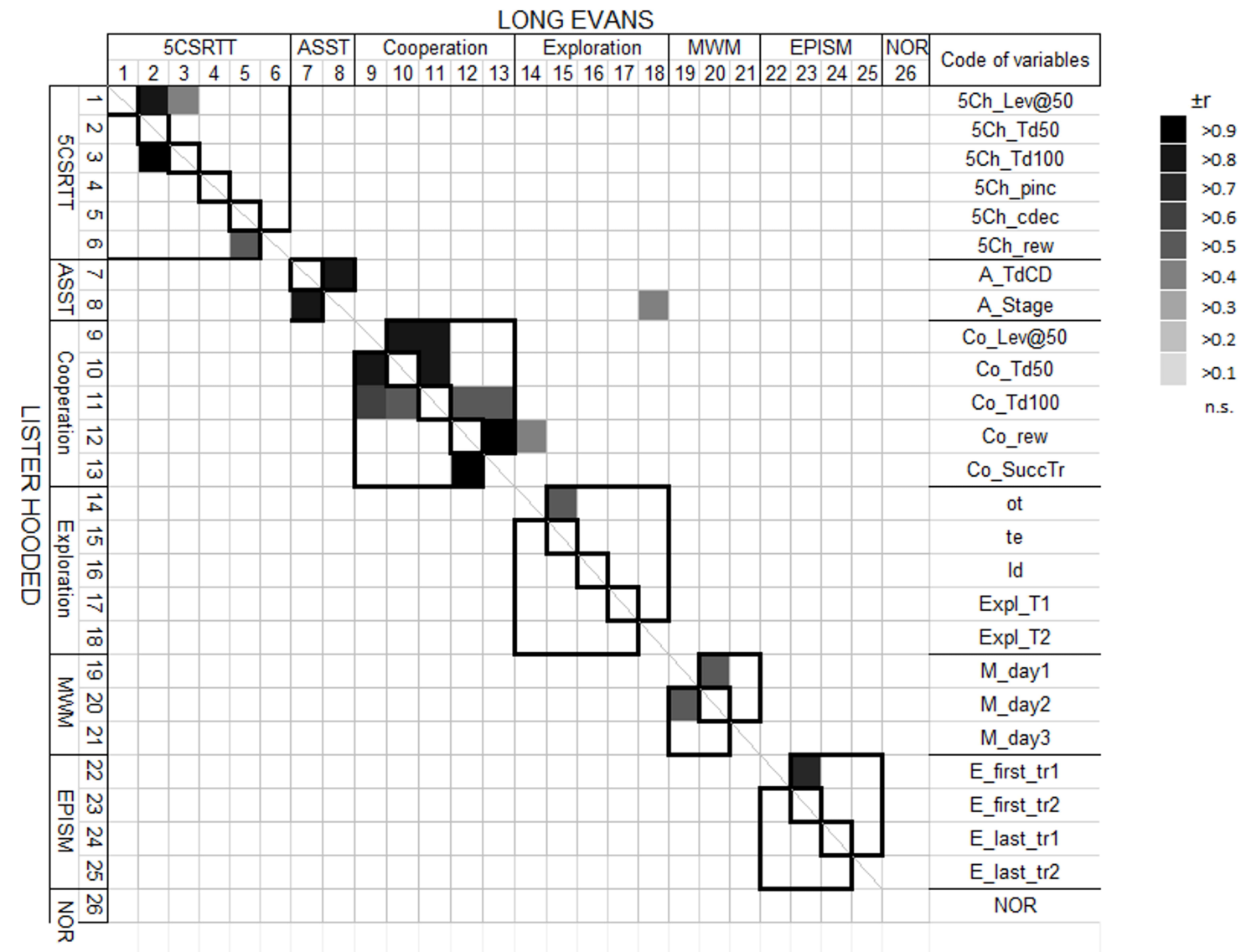

Fig. 2. Results of correlation analysis in Long-Evans (right top) and Lister Hooded rats (left bottom). Rows and columns represent variables of the raw datasets cells correspond to correlations. Areas bordered with black line indicate correlations between variables from the same behavioral task. Significant correlations $(p<0.05)$ are marked with gray, darkness of the cells represents the strength of correlation according to the scale in the right upper corner. For detailed correlation matrices see Supplementary Fig. 1.

In the ASST, both parameters correlated in both strains (LE: $r=-0.83$, LH: $r=-0.86$ ).

Correlation patterns in the cooperation paradigm were also similar in the strains. All learning speed parameters correlated in both species. Training days required to complete the half and all of the training stages positively correlated (LE: $r=0.85$, LH: 0.53 ), while the stage reached when the population mean best approximated 50\% negatively correlated with these variables (days required to complete half of the training: $r=-0.88$ and -0.84 , days required to complete the entire training: $r=-0.84$ and -0.64 in LE and LH respectively). The two variables measuring performance, number of rewards obtained and proportion of successful trials, also showed a strong correlation (LE: $r=0.99$, LH: $r=0.98$ ). In LE strain days required to complete the training negatively correlated with the performance describing variables $(r=-0.55$ in both cases).

Among the variables composing the exploration variable, only one significant correlation was detected between the two EPM variables in LE rats $(r=0.51)$.

Escape latencies on the first and second day of Morris water-maze training significantly correlated in both strains (LE: $r=0.57$, LH: $r=0.51$ ).
In the episodic memory task, significant correlation was found only between trial latencies of the first part of the training in LE rats $(r=0.71)$.

In case of both strains, PCA did not effectively reduce the number of dimensions. According to the cross validation procedure, only the first components were significantly relevant in the model. The first two components explained only $30 \%$ and $25 \%$ of the total variance in LE and LH rats, respectively. Variables were represented by low power values in both species (Fig. 3A,B) and few of them were considered as relevant in the PCAs. In both strains, variables of the COOP test showed high loads along the first component: training days required to complete the half or entire training located in opposite direction with the stage reached when the entire population reached the half of the training and with the two performance parameters (proportion of successful trials and number of collected rewards) indicating a negative relationship between learning speed and success in collecting reward (Fig. 3C,D). Along the second component, 5CSRTT learning parameters displayed higher load (time required to complete the half and entire training in LE and LH rats, respectively). 

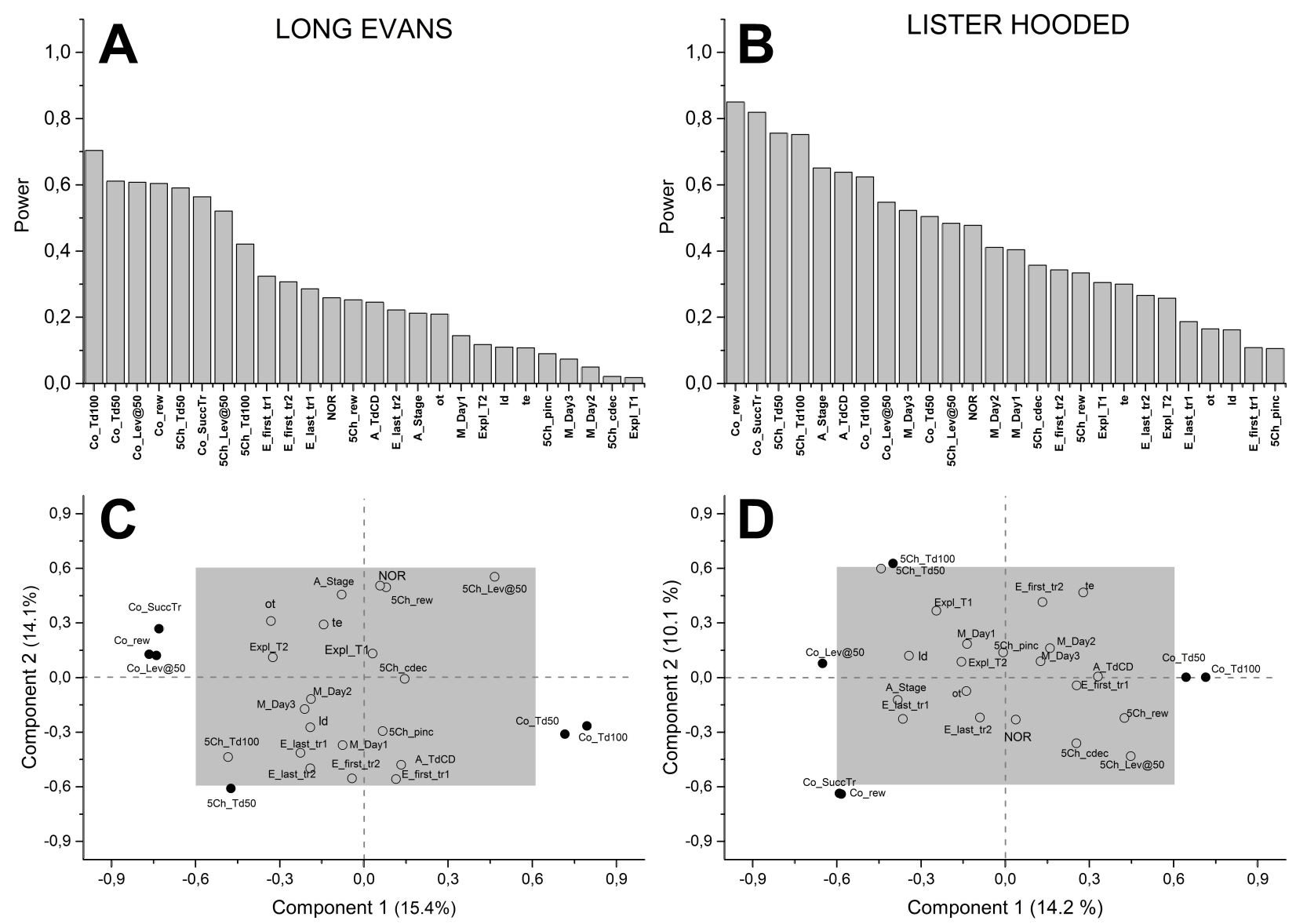

Fig. 3. Results of PCAs of the raw variable data. On panel A and B, power of variables in PCA models are displayed for the two rat strains. Power ranges from 0 to 1 . If the power of a variable reaches 1 , its entire variance is explained by the model. On panel C and D, variables are displayed on scatterplots according to their loadings along the two retained components of the PCAs. Percentage values after the component names indicate the variance explained by the component. Gray squares indicate the space, where variable loads are less than 0.6 along the components, either in negative or in positive directions. Variables that fall outside this range are regarded as relevant and marked with black points. For variable codes see Tables 1,2 .

\subsection{Relation of composite variables}

In LH rats, significant correlation was not detected among the composite variables. In case of LE rats, only one relatively low significant correlation was detected between NOR and EPISM ( $r=0.40)$, however by removing one outlier point from the analysis the correlation ceases (for details see Supplementary Figs. 1,2).

Similarly to the raw variables, only the first component had significant relevance in the PCA in case of both strains. The first two components also explained only a minor portion, $46 \%$ and $42 \%$ of the total variance for LE and LH rats, respectively. Importance of variables were low in the models, especially in LH rats, where the power exceeded 0.6 only in case of one variable (Fig. 4A,B). In LE rats NOR and EPISM had high loads along the first component, while in the other strain, COOP and EXPL displayed high loads in the same direction along the first component. In contrast, in LE rats COOP showed high load along the second component, while ASST displayed high load along the 2 nd component in both strains (Fig. 4).

\subsection{Characterization of composite scores}

Composite scores had normal distribution in both strains. Variance was higher in LE rats compared to LH rats (Supplementary Fig. 4). In both strains, animals showed variable performance in the different behavioral tests (Fig. 5). Test efficacy was not completely uniform even in case of those individuals that displayed high or low overall performance (Fig. 5).

\section{Discussion}

\section{Cognitive functions measured in the different tasks are not related}

We could not detect a general cognitive factor that consistently determines the performance of animals in different cognitive tasks in any of the tested rat strains. Instead, our data show that individuals have divergent capabilities at different cognitive domains.

In the raw variable datasets, with the exception of a single, modest, hardly interpretable, probably "by-chance" correlation in the LE strain, significant correlations were only detected among variables describing the same paradigm. 

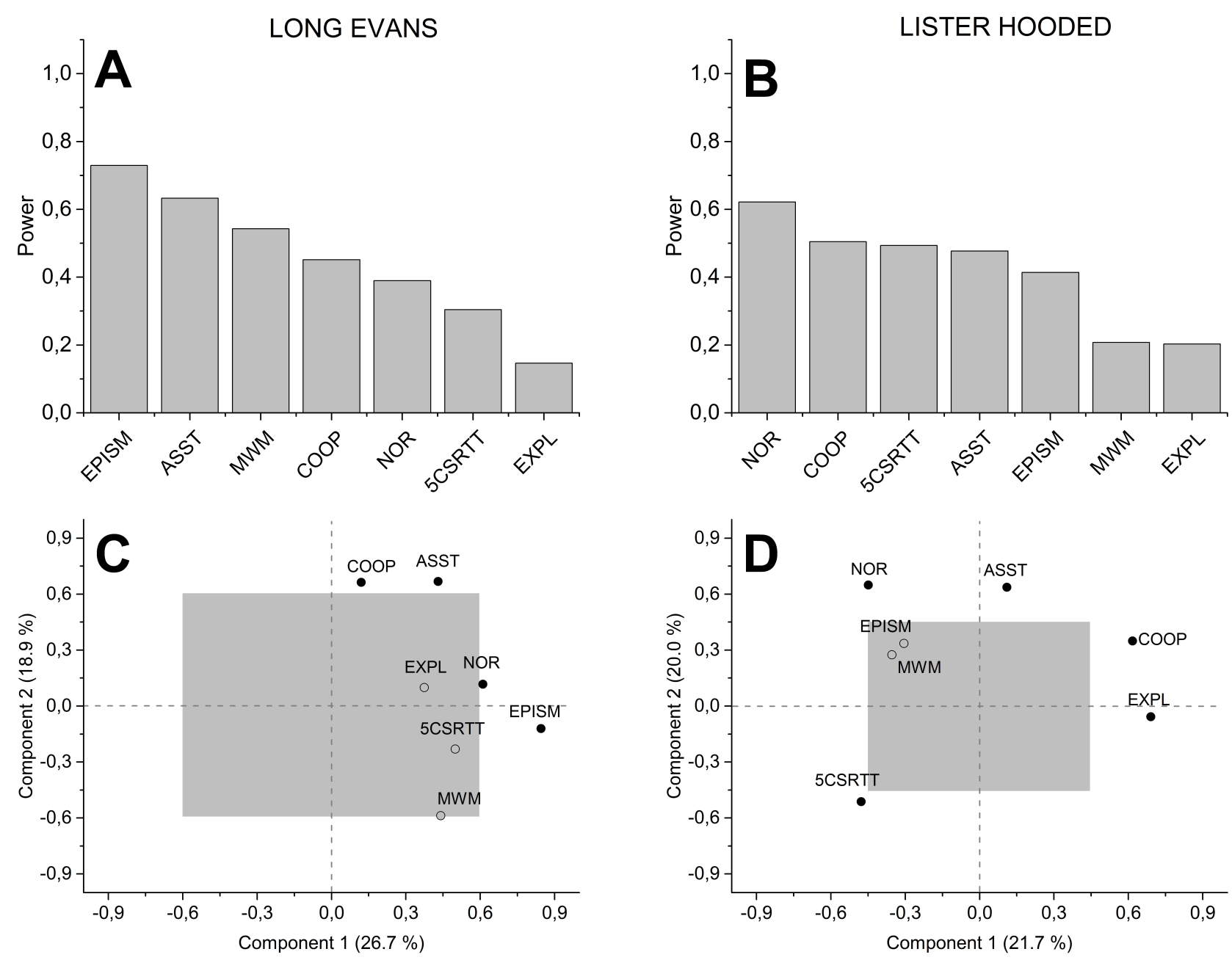

Fig. 4. Results of PCAs of the composite variable data. On panel A and B, power of variables in PCA models are displayed for the two rat strains. Power ranges from 0 to 1 . If the power of a variable reaches 1 , its entire variance is explained by the model. On panel C and D, variables are displayed on scatterplots according to their loadings along the two retained components of the PCAs. Percentage values after the component names indicates the variance explained by the component. Gray squares indicate the space, where variable loads are less than 0.6 along the components, either in negative or in positive directions. Variables that fall outside this range are regarded as relevant and marked with black points.

Within this subset, the majority of the correlations were observed between learning speed variables; task performance variables correlated much less either with each other or with learning speed variables. PCAs did not reduce the dimensionality of the raw variables, which also indicates these represent strongly heterogeneous functions. In both strains, variables of COOP showed high loads on the first component, while 5CSRTT learning parameters (5Ch_Td50 and 5Ch_Td100 in $\mathrm{LE}$ and $\mathrm{LH}$, respectively) stood out along the second axis. It may simply reflect the fact that these two tests were represented with the highest number of variables, and thus these assays showed the highest number of significant within-test correlations.

Independence of composite variables describing overall performance in a particular test also suggests that the applied behavioral paradigms measure different types of cognitive functions. Like in case of the raw variables, PCAs could not effectively reduce data dimensionality. Along the first com- ponent, EPISM and NOR were the prominent variables in LE rats, whereas in LH rats COOP and EXPL had high loads on the first component. Along the second component, ASST showed high loads in both species accompanied by COOP in the LE and NOR in the LH strain. The lack of linear correlations among the variables in both strains, and the low level of variance explained by the PCAs indicate that location of variables in the PCA plot is practically accidental, and this can be the source of the observed strain differences.

The composite score includes all the domains represented by the different tasks, thus it can be assumed that it describes the overall performance of the animals well. The normal distribution of composite scores confirms this assumption, as the two animal groups can be considered as randomly selected populations. Normality was also found in case of another, differently calculated variable describing overall performance in a cognitive test battery in mice [24]. Higher variance in case of LE rats may have resulted from the wider time win- 

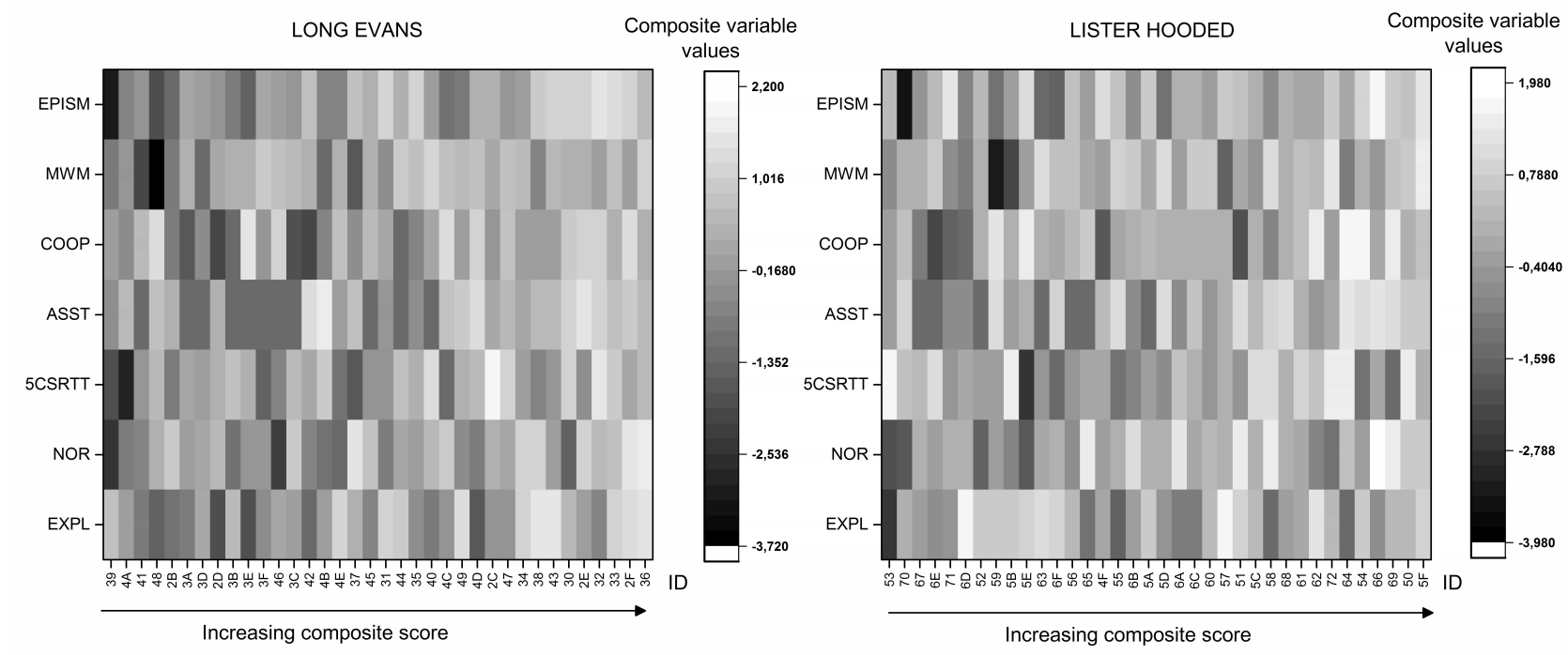

Fig. 5. Composite variable values of the different tests as a function of composite scores. On the heat maps each column represents an individual, ID indicates the identification code of the animal, rows represent variables describing test performance. Individuals are ranked in increasing order from the left to the right based on their composite scores. (Animal ID-s on the $\mathrm{X}$ axis from left to right: Long-Evans: 39, 41, 48, 4A, 3E, 2B, 3C, 3A, 2D, 3B, 4B, 42, 40, 46, 37, 45, 3F, 3D, 4E, 4D, 35, 49, 31, 44, 4C, 43, 2C, 38, 30, 47, 34, 32, 2E, 33, 2F, 36. Lister Hooded: 53, 70, 6E, 5B, 71, 5E, 67, 52, 6D, 65, 6F, 6B, 4F, 56, 6C, 59, $55,63,5 \mathrm{~A}, 5 \mathrm{D}, 57,6 \mathrm{~A}, 51,5 \mathrm{C}, 54,61,64,60,68,62,66,58,72,69,50,5 \mathrm{~F})$.

dow when the tests were performed. Variable performance of high and low scored animals in different assays also indicates that individual success in a behavioral task is rather assay-specific than determined by a general learning ability factor.

The absence of $g$ factor in our study contrasts with the majority of rodent literature. A possible source of the discrepancy may have resulted from the used animal population. Most of the studies where ' $\mathrm{g}$ ' was detected were conducted in mice. These studies come from two labs. The Matzel group conducted experiments in CD1 mice [20-24] applying spatial navigation (maze) paradigms as well as aversive conditioning and odor discrimination. They repeatedly found a $g$ factor identified as the first principal component accounting for 30$40 \%$ of the total variance and with positive and considerable (0.2-0.8) factor loadings of all the cognitive assays. They got similar results with inbred Balb/C strain, too [25]. Galsworthy et al. [18] studied a heterogeneous stock mouse line in a variety of problem solving and spatial navigation tasks and reported a $g$ factor which accounted for $30 \%$ of the variance. However, when additional learning assays were included, $g$ factor only accounted for $18 \%$ of the total variance and the factor loadings also became lower [19]. In contrast, Locurto et al. [31] could not identify a $g$ factor satisfying the above conditions $(-30 \%$ of variance, positive and notable loading of all cognitive variables) in the same strain in a set of spatial and working memory tasks. Much less data are available in the rat. $g$ factor was detected in Long-Evans rats, and it correlated with brain weight [17]. Thorndike [32] also found correlations among performance levels of albino rats in different tasks, although those were stronger among maze learning and problem solving parameters than among conditioned avoidance tasks and maze learning or problem solving. However, $g$ factor was not found in Sprague-Dawley rats tested in a set of maze-learning, detour and discrimination paradigms [33]. Factor analysis in a recent study showed that distinct behavioral abilities and traits, such as spatial cognition, anxiety- and depression-related behavior, motor abilities and activity well separated along different components [34]; however, in this battery only one cognitive test was used. Nevertheless, due to the low number of rat studies, it is not possible to make a meaningful comparative analysis for the species.

Our statistical methodology may have also contributed to detecting a higher level of independence among the performances in different tasks compared to earlier studies. To reduce false discovery rate (which is a basic statistical requirement in case of multiple comparison), in the correlation analysis we corrected the significance level with sequential Bonferroni adjustment according to the number of correlations calculated. This correction of significance levels was not applied (or, at least, not described) in the above cited rodent studies, which may have resulted in detecting a higher number of significant correlations in the datasets than that they contained in reality (see e.g., [35]).

It also has to be taken into account, that the cognitive tasks were executed at different ages of the animals. It is certainly a deficiency of the study but it has to be noted that our basic aim was to create a rat population with widespread knowledge suitable for further experiments with cognitive enhancer drugs [3]. Therefore the study was not specifically designed for detecting a $g$ factor neither for doing a strict head-to-head comparison of the two rat strains. This 
age heterogeneity may have decreased the possibility to detect correlations among the variables as cognitive abilities may have changed with aging. However, food restriction increases longevity of rodents [36, 37], therefore we assume that age-related cognitive decline may have been less prominent in our rats, than in ad libitum fed animals. In fact, group means of COOP performance parameters show that learning capabilities of the animals were maintained up to 2 years of age. It is of note that there was no meaningful difference in the performance of Long-Evans and Lister Hooded rats, notwithstanding that in some of the assays they were measured at different ages. Furthermore, considering the postulated global nature and trait character of the $g$ factor we assume if a general learning ability factor had existed in any of the two rat populations, it should have been detected independently of the timing of the paradigms.

A more important shortcoming of the study may be that it was only done in males (because of logistic reasons). Including females may have added more variance to the results thus may have increased the chance to detect a $\mathrm{g}$ factor.

Another deficiency of our test battery was the lack of working memory tasks. As working memory was reported to correlate with general cognitive abilities [20] and working memory training was shown to improve general cognitive performance in mice [38], investigating the relation of working memory tasks with the rest of the model set may have provided valuable information.

The apparent independence of the cognitive functions seen in the study could have been better judged if each cognitive domain had not been tested in a single model. Ideally, a domain should be tested at least in two models which differ in their non-cognitive demands (for example, MWM and 8arm radial maze with external cues when both tests measure allocentric spatial memory). This way, the non-cognitive source of behavioral variance can be discovered [19]. Such data can also provide information on the validity of the models (i.e., lack of correlation between models assumed to test the same cognitive domain may indicate that one or both of the models measure something else) as well as on their true independence (i.e., models of the same cognitive domain should correlate with each other whereas lack of correlation should occur between models of unrelated cognitive functions).

\section{Conclusions}

In conclusion, in the applied test battery a general cognitive ability factor was not detected, and the performance of animals in the different cognitive models were independent from each other. This finding was replicated in two different rat strains. It may have resulted from the assays covered distinct cognitive domains, which had been an aim in designing our test battery. Investigating whether it is possible to selectively impair and improve performance at different cognitive domains will provide information on the validity of this assumption. The results also point out that correlational and principal component analyses may not only be helpful in revealing a $g$ factor but also in controlling the translational validity of an animal test battery intended to predict human efficacy.

\section{Abbreviations}

ASST, attentional set shifting task; COOP, cooperation task; 5-CSRTT, 5-choice serial reaction time task; EDS, extra dimensional shift; EPISM, episodic memory test; EPM, elevated plus-maze; EXPL, exploratory inclination; IDS, intra dimensional shift; LE, Long-Evans; LH, Lister Hooded; MWM, Morris water-maze test; NOR, novel object recognition test; PCA, principal component analysis; PJT, pot jumping test.

\section{Author contributions}

FK designed and performed the experiments, analyzed and interpreted the data, conceived and performed the statistical analyses and wrote the paper. KK, IP designed and performed the experiments, analyzed and interpreted the data. AJE designed and performed the experiments, analyzed and interpreted the data and reviewed and edited the manuscript. IG conceived and designed the experiments, analyzed and interpreted the results, wrote and reviewed the paper and supervised the work.

\section{Ethics approval and consent to participate}

Housing and all procedures carried out on animals were authorized by the regional animal health authority in Hungary (resolution number PEI/001/3572-4/2014) and conformed to the Hungarian welfare legislation, ARRIVE guidelines and the EU 63/2010 Directive.

\section{Acknowledgment}

We thank Kerekesné Csontos Ildikó for her valuable technical assistance.

\section{Funding}

This work was supported by the Hungarian National Brain Research Program (NAP) [KTIA_NAP_13-20140015] and supported by the Higher Education Institutional Excellence Programme of the Ministry of Human Capacities in Hungary, within the framework of the Neurology thematic programme of the Semmelweis University (FIKP 2018).

\section{Conflict of interest}

The authors declare no conflict of interest.

\section{Supplementary material}

Supplementary material associated with this article can be found, in the online version, at https://www.imrpress.com/j ournal/JIN/21/1/10.31083/j.jin2101012. 


\section{References}

[1] DiLuca M, Olesen J. The cost of brain diseases: a burden or a challenge? Neuron. 2014; 82: 1205-1208.

[2] Bespalov A, Steckler T. Lacking quality in research: is behavioral neuroscience affected more than other areas of biomedical science? Journal of Neuroscience Methods. 2019; 300: 4-9.

[3] Gyertyán I. Cognitive 'Omics': Pattern-Based Validation of Potential Drug Targets. Trends in Pharmacological Sciences. 2017; 38: 113-126.

[4] Gyertyán I. How can preclinical cognitive research further neuropsychiatric drug discovery? Chances and challenges. Expert Opinion on Drug Discovery. 2020; 15: 659-670.

[5] Garner JP. The significance of meaning: why do over $90 \%$ of behavioral neuroscience results fail to translate to humans, and what can we do to fix it? ILAR Journal. 2015; 55: 438-456.

[6] Millan MJ, Agid Y, Brüne M, Bullmore ET, Carter CS, Clayton NS, et al. Cognitive dysfunction in psychiatric disorders: characteristics, causes and the quest for improved therapy. Nature Reviews. Drug Discovery. 2012; 11: 141-168.

[7] Martis L, Krog S, Tran TP, Bouzinova E, Christiansen SL, Møller A, et al. The effect of rat strain and stress exposure on performance in touchscreen tasks. Physiology \& Behavior. 2018; 184: 83-90.

[8] Harker KT, Whishaw IQ. Place and matching-to-place spatial learning affected by rat inbreeding (Dark-Agouti, Fischer 344) and albinism (Wistar, Sprague-Dawley) but not domestication (wild rat vs. Long-Evans, Fischer-Norway). Behavioural Brain Research. 2002; 134: 467-477.

[9] Robbins TW. The 5-choice serial reaction time task: behavioural pharmacology and functional neurochemistry. Psychopharmacology. 2003; 163: 362-380.

[10] Birrell JM, Brown VJ. Medial frontal cortex mediates perceptual attentional set shifting in the rat. The Journal of Neuroscience. 2000; 20: 4320-4324.

[11] Ennaceur A, Delacour J. A new one-trial test for neurobiological studies of memory in rats. 1: Behavioral data. Behavioural Brain Research. 1989; 31: 47-59.

[12] Morris R. Developments of a water-maze procedure for studying spatial learning in the rat. Journal of Neuroscience Methods. 1984; 11: 47-60.

[13] Kozma K, Kassai F, Ernyey AJ, Gyertyán I. Establishment of a rodent cooperation assay as a model of social cognition. Journal of Pharmacological and Toxicological Methods. 2019; 97: 44-51.

[14] Ernyey AJ, Grohmann Pereira T, Kozma K, Kouhnavardi S, Kassai F, Gyertyán I. Following of aging process in a new motor skill learning model, "pot jumping" in rats. GeroScience. 2019; 41: 309319.

[15] Ernyey AJ, Bogi E, Kassai F, Plangár I, Gyertyán I. Translational difficulties in querying rats on 'orientation'. BioMed Research International. 2019.

[16] Pellow S, Chopin P, File SE, Briley M. Validation of open:closed arm entries in an elevated plus-maze as a measure of anxiety in the rat. Journal of Neuroscience Methods. 1985; 14: 149-167.

[17] Anderson B. Evidence from the rat for a general factor that underlies cognitive performance and that relates to brain size: intelligence? Neuroscience Letters. 1993; 153: 98-102.

[18] Galsworthy MJ, Paya-Cano JL, Liu L, Monleón S, Gregoryan G, Fernandes C, et al. Assessing reliability, heritability and general cognitive ability in a battery of cognitive tasks for laboratory mice. Behavior Genetics. 2006; 35: 675-692.

[19] Galsworthy MJ, Paya-Cano JL, Monleón S, Plomin R. Evidence for general cognitive ability $(\mathrm{g})$ in heterogeneous stock mice and an analysis of potential confounds. Genes, Brain, and Behavior. 2003; 1: 88-95.

[20] Kolata S, Light K, Grossman HC, Hale G, Matzel LD. Selective attention is a primary determinant of the relationship between working memory and general learning ability in outbred mice. Learning \& Memory. 2019; 14: 22-28.

[21] Kolata S, Light K, Townsend DA, Hale G, Grossman HC, Matzel LD. Variations in working memory capacity predict individual differences in general learning abilities among genetically diverse mice. Neurobiology of Learning and Memory. 2005; 84: 241-246.

[22] Light KR, Kolata S, Hale G, Grossman H, Matzel LD. Upregulation of exploratory tendencies does not enhance general learning abilities in juvenile or young-adult outbred mice. Neurobiology of Learning and Memory. 2008; 90: 317-329.

[23] Light KR, Grossman H, Kolata S, Wass C, Matzel LD. General learning ability regulates exploration through its influence on rate of habituation. Behavioural Brain Research. 2011; 223: 297-309.

[24] Matzel LD, Han YR, Grossman H, Karnik MS, Patel D, Scott N, et al. Individual Differences in the Expression of a "General" Learning Ability in Mice. The Journal of Neuroscience. 2003; 23: 64236433.

[25] Matzel LD, Grossman H, Light K, Townsend D, Kolata S. Agerelated declines in general cognitive abilities of Balb/C mice are associated with disparities in working memory, body weight, and general activity. Learning \& Memory. 2009; 15: 733-746.

[26] Warne RT, Burningham C. Spearman'sg found in 31 nonWestern nations: Strong evidence that $\mathrm{g}$ is a universal phenomenon. Psychological Bulletin. 2019; 145: 237.

[27] Gyertyán I, Plangár I, Ernyei A, Császár E, Kozma K, Kassai F. Developing a rodent test battery for characterizing cognitive enhancer drug-candidates. European Neuropsychopharmacology. 2016; 26: S341-S342.

[28] Motulsky H. Intuitive biostatistics. Oxford University Press Ltd.: New York, USA. 1995

[29] Dell Inc. PCA Example. 2016. Available at: http: //documentation.statsoft.com/STATISTICAHelp.aspx?pat h=MSPC/PCA/PCAExample (Accessed: 25 January 2019).

[30] Osborne JW, Costello AB. Sample size and subject to item ratio in principal components analysis. Practical Assessment, Research \& Evaluation. 2004; 9:11.

[31] Locurto C, Fortin E, Sullivan R. The structure of individual differences in heterogeneous stock mice across problem types and motivational systems. Genes, Brain, and Behavior. 2003; 2: 40-55.

[32] Thorndike RL, Organization of behavior in the albino rat. Genetic Psychology Monographs. 1935; 17: 1-70

[33] Thompson R, Crinella FM, Yu J. Brain Mechanisms in Problem Solving and Intelligence: a Lesion Survey of the Rat Brain. Critical Issues in Neuropsychology. Plenum Press: New York and London. 1990.

[34] Feyissa DD, Aher YD, Engidawork E, Höger H, Lubec G, Korz V. Individual Differences in Male Rats in a Behavioral Test Battery: a Multivariate Statistical Approach. Frontiers in Behavioral Neuroscience. 2019; 11: 26.

[35] Arendash GW, King DL. Intra- and intertask relationships in a behavioral test battery given to Tg2576 transgenic mice and controls. Physiology \& Behavior. 2002; 75: 643-652.

[36] Bagherniya M, Butler AE, Barreto GE, Sahebkar A. The effect of fasting or calorie restriction on autophagy induction: a review of the literature. Ageing Research Reviews. 2019; 47: 183-197.

[37] Harrison DE, Archer JR, Astle CM. Effects of food restriction on aging: separation of food intake and adiposity. Proceedings of the National Academy of Sciences of the United States of America. 1984; 81: 1835-1838.

[38] Light KR, Kolata S, Wass C, Denman-Brice A, Zagalsky R, Matzel LD. Working memory training promotes general cognitive abilities in genetically heterogeneous mice. Current Biology. 2011; 20: $777-782$. 